\section{Does screening for prostate cancer reduce prostate cancer mortality?}

To the Editor: Two large randomized controlled studies evaluating the efficacy of prostate cancer screening with prostate-specific antigen (PSA) testing were recently published in the New England Journal of Medicine. These landmark studies received a great deal of media attention and warrant some clarification. One was a US-based study of 76693 men from the Prostate, Lung, Colorectal and Ovarian (PCLO) Cancer Screening trial, ${ }^{1}$ and the other was the European Randomized Study of Screening for Prostate Cancer (ERSPC) of 162243 men. ${ }^{2}$ Subjects were randomly assigned to either PSA screening (in addition to prostate examinations) or no screening (control group). Using cancer-specific mortality as the primary end point, after about 10 years of follow-up, the PLCO study did not find any difference in mortality rates between the screening and control groups. In contrast, the ERSPC study found a $20 \%$ reduction in prostate cancer deaths in the screening group, but was associated with a high risk of overdiagnosis.

Although the conclusions of these studies appear to be negative or mixed, careful analysis leads to a more positive view. As PSA screening is widespread in Canada, ${ }^{3}$ it is important to clarify these issues.

In the PCLO study, only 94 prostate cancer-related deaths occurred (50 in the screening group and 44 in the control group, risk ratio $1.13,95 \%$ confidence interval [CI] 0.75-1.70). Critically, there was insufficient power to compare death rates for the followup period. Further, up to $52 \%$ of patients in the control group actually underwent PSA testing during the study period. Prostate-specific antigen screening clearly results in earlier diagnosis. Thus, in the control group, a higher proportion of men with more locally advanced cancers would be expected compared with the screening group. This was not observed. Indeed, the distribution of clinical stage among the patients diagnosed with prostate cancer between the screening and control groups were very similar. The proportion of advanced cancer (stage 3 or 4) was $3.5 \%$ in the screening group and $3.8 \%$ in the controls. The only subgroup that had a higher proportion of advanced cancer $(5.7 \%)$ was the patients in the screening group who had never been previously screened and diagnosed at the time of initial PSA screening.

The authors argued that the effect of contamination of PSA testing in the control group was not significant for 2 reasons - the estimated contamination rate was anticipated from the trial design, and the number of prostate cancer cases in the control group remained consistently lower than the screening group. However, screening outside the protocol in the control group was assessed by random surveys. Only a small proportion was examined $(1 \%$ every 1-2 yr). The rate of contamination of PSA testing in the control group may be underestimated. Also, examination of the cumulative rate of prostate cancer incidence curves in each of the arms was constant and did not separate. Over the study period, there was a consistent difference of about 500 prostate cancer cases between the screening and control arms. The number of cases diagnosed during the baseline screen within the screening arm was 549. After accounting for this difference, the rate of prostate cancer diagnoses was similar in both arms. Thus, since the control arm was significantly contaminated with patients who underwent PSA testing outside the study protocol, further follow-up is unlikely to show a difference between the groups.

In the ERSPC study, a higher number of deaths occurred compared with the PCLO study. There were 540 prostate cancer-related deaths, 214 in the screening group and 326 in the control group, with a risk ratio of $0.80(95 \%$ Cl 0.67-0.95, $p=0.01)$. Unfortunately, the rate of PSA testing in the control group was not specified. However, the proportion of patients diagnosed with locally advanced cancers in the control group (15.7\%) was 2 times higher than the screening group (8.6\%) (Supplementary Appendix of paper). This outcome is consistent with the stage migration associated with PSA screening, and reflects a meaningful difference in screening rates between the 2 arms (in contrast to the PLCO study). This is critical.

The authors cautioned that 48 additional subjects would need to be treated to prevent 1 death from prostate cancer, raising the concern of overdiagnosis. These calculations were based on the whole study period. However, the cumulative risk of death curves starts to diverge at 10 years and separate widely beyond 12 years after randomization. It is reasonable to expect that this trend would continue with further follow-up, meaning that the number needed to treat would continue to fall, increasingly favouring screening.

Based on these analyses, the PCLO study is inconclusive, while the ERSPC study shows a clear survival advantage in favour of prostate cancer screening. The magnitude of this benefit will become better defined with further follow-up. Also, the approach of active surveillance ${ }^{4}$ which is increasingly being adopted for favourable risk prostate cancer in Canada, would be expected to significantly reduce the number needed to treat for each death avoided. The ERSPC study, in conjunction with the Scandinavian trial demonstrating the benefit of radical prostatectomy compared with watchful waiting, ${ }^{5}$ clearly supports a benefit of early detection and treatment. 
Nevertheless, screening involves tradeoffs, and these risks and benefits remain an important point for discussion and education between physicians and patients.

\section{Robert K. Nam, MD, FRCSC}

Associate Professor of Surgery, Division of Urology, Sunnybrook Health Sciences Centre, University of Toronto, Toronto, Ont.

\section{Laurence H. Klotz, MD, FRSC}

President, Canadian Urological Association;

Professor of Surgery, Division of Urology,

Sunnybrook Health Sciences Centre, University of Toronto, Toronto, Ont.

Competing interests: None declared.

\section{References}

1. Andriole GL, Grubb RL III, Buys SS, et al. Mortality results from a randomized prostate-cancer screening trial. N Engl J Med 2009;360:1310-9.

2. Schroder FH, Hugosson J, Roobol MJ, et al. Screening and prostatecancer mortality in a randomized European study. $N$ Engl J Med 2009;360:1320-8.

3. Nam RK, Klotz LH. Trends in prostate cancer screening: Canada. In: Ankerst DP, Tangen CM, Thompson IM, Eds. Prostate cancer screening. 2nd ed. New York: Humana Press; 2009. p. 23-35.

4. Klotz L. Active surveillance for prostate cancer: For whom? J Clin Oncol 2005;23:8165-9.

5. Bill-Axelson A, Holmberg L, Ruutu M, et al. Radical prostatectomy versus watchful waiting in early prostate cancer. N Engl J Med 2005;352:1977-84.

\section{CUA PATIENT INFORMATION BROCHURES}

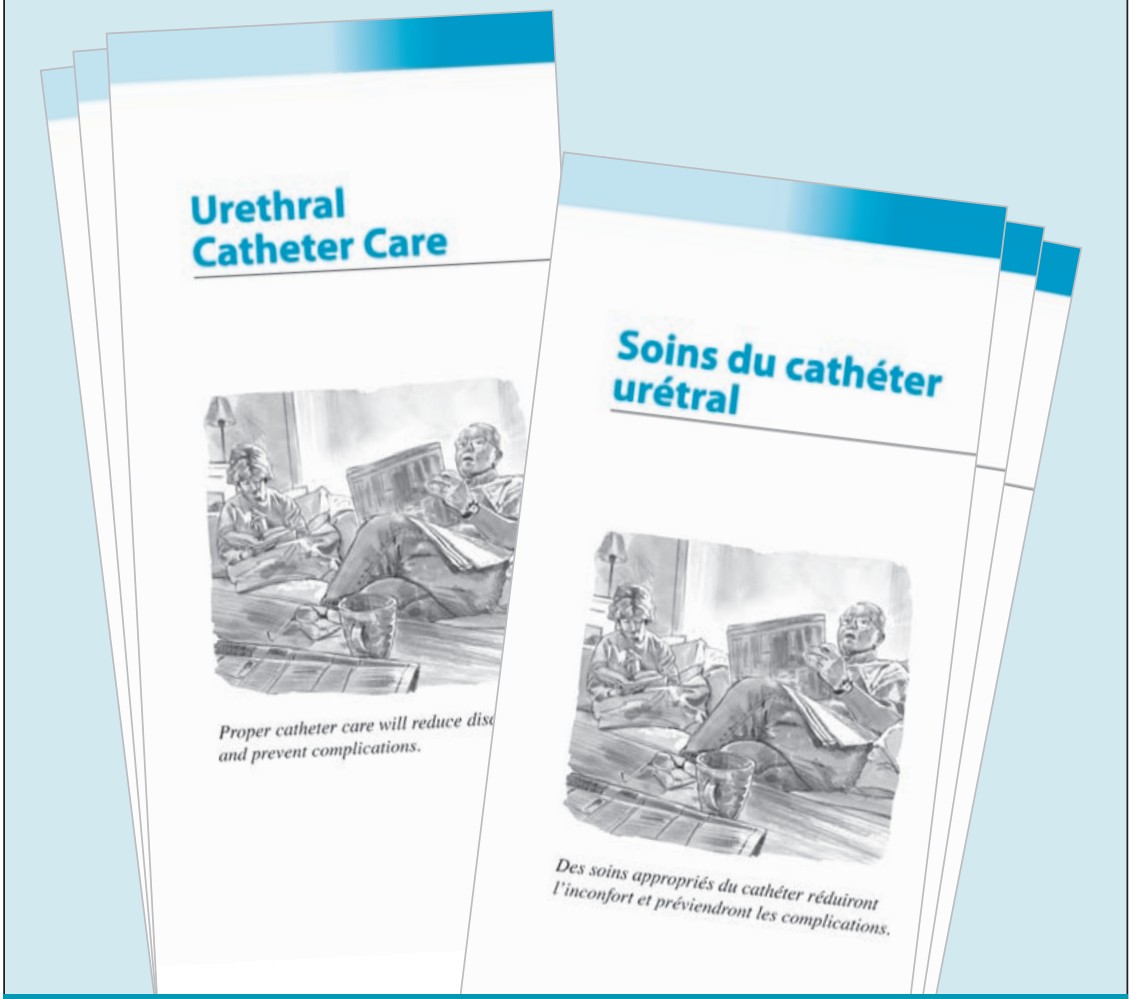

- For more information on CUA Patient Information Brochures, see page 253.

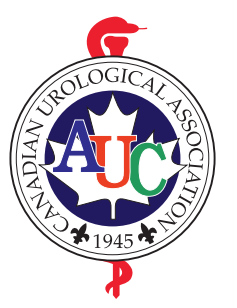

